\title{
The ATM-dependent DNA Damage Signaling Pathway
}

\author{
R. KITAGAWA* AND M.B. KaStAN \\ Department of Hematology-Oncology, St. Jude Children's Research Hospital, \\ Memphis, Tennessee 38105
}

\begin{abstract}
Many of the insights that we have gained into the mechanisms involved in cellular DNA damage response pathways have come from studies of human cancer susceptibility syndromes that are altered in DNA damage responses. ATM, the gene mutated in the disorder, ataxia-telangiectasia, is a protein kinase that is a central mediator of responses to DNA double-strand breaks in cells. Recent studies have elucidated the mechanism by which DNA damage activates the ATM kinase and initiates these critical cellular signaling pathways. The SMC1 protein appears to be a particularly important target of the ATM kinase, playing critical roles in controlling DNA replication forks and DNA repair after the damage. A major role for the NBS1 and BRCA1 proteins appears to be in the recruitment of an activated ATM kinase molecule to the sites of DNA breaks so that ATM can phosphorylate SMC1. Generation of mice and cells that are unable to phosphorylate SMC1 demonstrated the importance of SMC1 phosphorylation in the DNA-damage-induced S-phase checkpoint, in determining rates of repair of chromosomal breaks, and in determining cell survival after DNA damage. Focusing on ATM and SMC1, the molecular controls of these pathways is discussed.
\end{abstract}

Cells from patients with the cancer-prone disorder, ataxia telangiectasia, exhibit many abnormalities in cellular DNA damage responses. For example, these cells have defective $\mathrm{G}_{1}$, intra-S-phase, and $\mathrm{G}_{2} / \mathrm{M}$ checkpoints and exhibit decreased survival and increased chromosomal breakage in response to ionizing irradiation (IR). These observations suggest that the gene product that is defective in this disorder, ATM (ataxia telangiectasia, mutated), is an important component of cellular responses to DNA damage. Results from numerous laboratories generated over the past decade since the gene was cloned have shed light on the central role that the ATM protein plays in DNA damage responses (Shiloh and Kas$\tan 2001$ ). In this paper, we discuss what is known about the molecular mechanisms by which the ATM protein kinase is activated by DNA damage, then follow with an overview of the substrates of ATM that have been implicated in cellular responses to DNA damage including a more extensive discussion of one particular substrate, $\mathrm{SMC1}$, that appears to be involved in influencing cell cycle progression, cell survival, and genetic integrity following DNA damage.

\section{ACTIVATION OF THE ATM PROTEIN KINASE}

Identification of the ATM gene by Shiloh and colleagues (Savitsky et al. 1995) revealed a very large gene that coded for a protein containing a phosphoinositide 3 (PI3) kinase-like sequence in its carboxyl terminus. Once it became technically feasible to generate wild-type and mutant full-length recombinant cDNAs and protein, we demonstrated that ATM was a bona fide protein kinase

*Present address: Department of Molecular Pharmacology, St. Jude Children's Research Hospital, 322 N. Lauderdale Street, Memphis, Tennessee 38105 . with no detectable lipid kinase activity (Canman et al. 1998). Following exposure of human cells to IR, the cellular activity of ATM increases (Banin et al. 1998; Canman et al. 1998). The observation that ATM itself was a phosphoprotein whose phosphorylation increased while performing in vitro kinase reaction assays (Canman et al. 1998) focused our attention on ATM phosphorylation as a potential contributor to its increased cellular activity during DNA damage responses. A site of DNA-damageinduced phosphorylation in this 3056-amino acid protein was found to be Ser-1981 by incubating cells with $\left.{ }^{[2} \mathrm{P}\right]$ orthophosphate, immunoprecipitating ATM protein, identifying one primary phosphopeptide during two-dimensional gel analysis after proteolytic degradation, followed by manual Edman degradation of proteolyzed peptides (Bakkenist and Kastan 2003). Ser-1981 is located in the amino terminus of the FAT domain (a domain shared by FRAP, ATM, and TRRAP) of ATM (Fig. 1A). The FAT domain is a region of approximately 500 amino acids that has some conservation among members of the PI3 kinase family of proteins (Bosotti et al. 2000).

We generated a rabbit polyclonal antibody specific for phosphorylated Ser-1981 in order to begin characterization of the functional role of this phosphorylation event. The kinetics and quantitation of the phosphorylation turned out to be quite extraordinary (Bakkenist and Kas$\tan 2003$ ). More than $50 \%$ of all cellular ATM is phosphorylated on Ser-1981 in less than 5 minutes after exposure to doses of IR as low as 0.5 Gy (Fig. 1B). The presence of phosphorylated ATM could be detected at doses as low as $0.1 \mathrm{~Gy}$, which should theoretically induce approximately four DNA strand breaks per cellular genome on average. Since ATM phosphorylation was also induced by the introduction of DNA strand breaks following transfection of the restriction enzyme I-Scel, ATM was responding to DNA breakage rather than to some other effect of irradiation on cells. Thus, ATM 
A

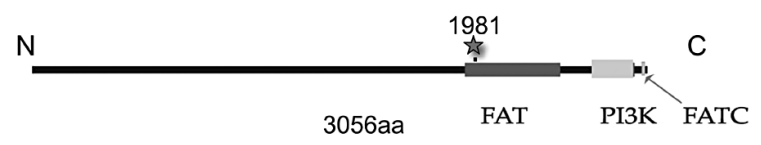

B

a

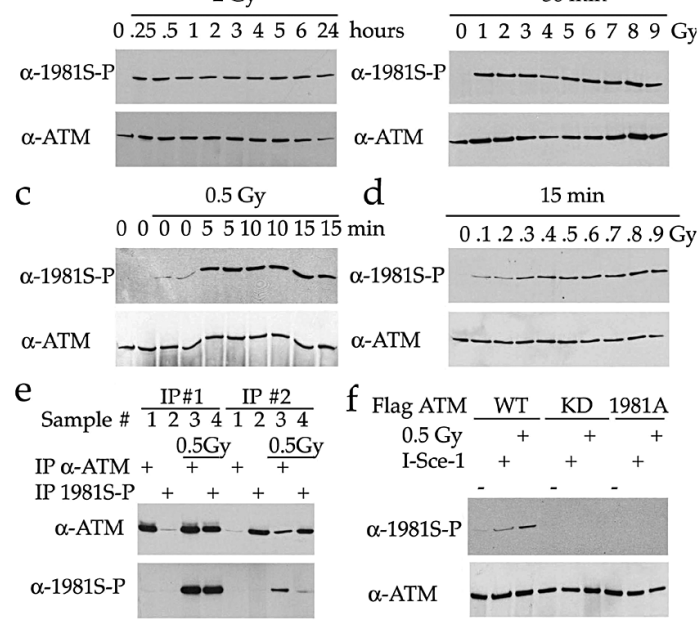

Figure 1. Autophosphorylation of the ATM kinase. (A) Schematic representation of the domains of ATM illustrating the approximate location of the Ser-1981 with the star. (B) Kinetics, stoichiometry, and detection sensitivity of ATM phosphorylation. $(a, b)$ Phosphorylation of Ser-1981 is maximal within $5 \mathrm{~min}$ after exposure to IR. Exponentially growing primary fibroblasts were exposed to 0.5 or 2 Gy IR. ATM immunoprecipitation was performed at the times indicated, and the levels of phosphorylation at Ser-1981 and total immunoprecipitated ATM were assessed by immunoblotting. $(c, d)$ Phosphorylation of Ser-1981 is maximal at about 0.4 Gy of IR. ATM was immunoprecipitated from exponentially growing primary fibroblasts $30 \mathrm{~min}$ after various doses of IR, and the levels of phosphorylation at Ser-1981 and total ATM were determined by immunoblotting. (e) Over $50 \%$ of ATM is phosphorylated within 15 min following irradiation at $0.5 \mathrm{~Gy}$. Exponentially growing primary fibroblasts were exposed to 0 or 0.5 Gy IR, and lysates were subjected to sequential immunoprecipitation with conventional anti-ATM or anti-ATMS1981p antibody followed by conventional anti-ATM antibody. The amount of ATM immunoprecipitated was determined by anti-ATM immunoblotting, and the specificity and completeness of ATMS1981p immunoprecipitation were confirmed by anti-ATMS1981p immunoblotting. ( $f$ ) The anti-ATMS1981p antibody can detect the presence of a few DNA breaks in the human genome. Constructs expressing Flag-tagged wild-type ATM, ATMkd, or ATMS1981A were cotransfected with a construct encoding the restriction enzyme I-Scel into GM00637 cells that had integrated two copies of a plasmid containing the sequence cut by IScel. As a control, ATM was immunoprecipitated from irradiated cells (0.5 Gy for $30 \mathrm{~min})$. Phosphorylation of Ser-1981 and total ATM were assessed by immunoblotting with antiATMS1981p or anti-Flag. (Reprinted, with permission, from Bakkenist and Kastan 2003 [@ Nature Publishing Group].)

phosphorylation after DNA damage was extraordinarily rapid, extensive, and sensitive.

The next question to ask was what kinase phosphorylated ATM following IR. Although an unbiased schema was used to identify the phosphorylation site, the Ser1981 site was a perfect ATM kinase consensus target (Kim et al. 1999). Thus, it was likely that ATM was either phosphorylated by a family member, such as ATR or DNA-PK, with similar target recognition, or we were observing an autophosphorylation event. Using a variety of approaches, we were able to show that the IR-induced phosphorylation required ATM kinase activity and that this was indeed an autophosphorylation event (Bakkenist and Kastan 2003).

To determine the physiological role of ATM S1981 phosphorylation, we generated an ATM mutant in which the Ser-1981 residue was replaced with alanine (ATMS1981A) so that IR-induced phosphorylation could not occur. Initially, we observed that the ATMS1981A mutant had normal in vitro kinase activity (Bakkenist and Kastan 2003), a result that seemed disappointing since it suggested that this phosphorylation event was not involved in the activation of the ATM kinase following IR. However, the ATMS1981A mutant had no activity in cells, since it failed to complement AT cells and had dominant-inhibitory effects in irradiated cells containing normal endogenous ATM. The reason for this apparent discrepancy between the in vitro and in vivo activities of the ATM S19181A mutant became clear when we observed that an isolated ATM kinase domain bound tightly to a fragment of ATM containing the ATM autophosphorylation site (amino acid residues 1961-2046) in Escherichia coli. If S1981 was mutated to alanine, the kinase domain still bound to this fragment, but if this serine was mutated to phosphorylation-mimic mutants such as aspartic acid or glutamic acid, the kinase domain could no longer bind to the S1981 domain. These observations led to a model where the kinase domain of ATM binds to this internal domain of ATM containing Ser-1981, but only when the serine residue is not phosphorylated. This could occur either because ATM exists in the cell as a homodimer or because the kinase domain folds over and binds to the S1981 domain in the same ATM molecule. We went on to demonstrate that ATM is present in unirradiated cells as a homodimer and that exposure of cells to IR resulted in a dissociation of this dimer. If one molecule of the dimer could not be phosphorylated on S1981, either because one molecule was mutated to be kinase-dead or because the S1981 site was mutated to alanine, then IR did not cause a dissociation of the dimer. This observation, combined with the autophosphorylation results, led to a model in which ATM is inactive in unstressed cells because the kinase domain is blocked by its binding to the internal S1981 domain and IR induces an intermolecular autophosphorylation event that causes dissociation of the ATM homodimer and release of an active ATM kinase.

Although the mechanism by which cellular ATM activity was increased following DNA damage was explained by this model, it remained unclear why intermolecular autophosphorylation of ATM was initiated following the introduction of DNA strand breaks. We reasoned that ATM might not need to go to the DNA strand break itself in order to be activated. This concept arose from the fact that ATM was activated so rapidly and 


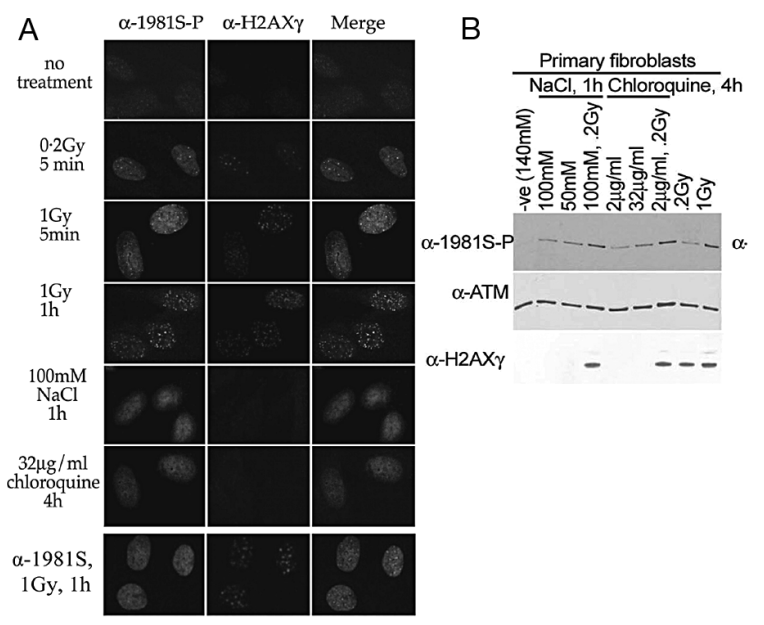

C

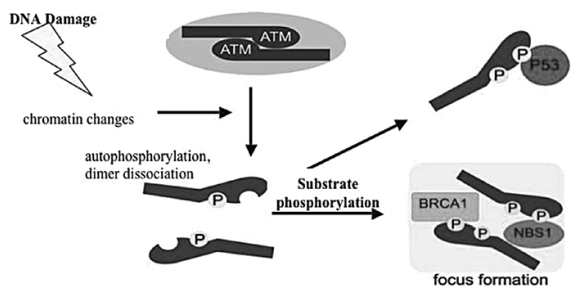

Figure 2. Activation of the ATM kinase independent of binding to DNA DSBs. (A) Primary fibroblasts grown on glass slides were irradiated $(0.2$ or $1 \mathrm{~Gy})$ and allowed to recover for $5 \mathrm{~min}$ or $1 \mathrm{hr}$, incubated in hypotonic buffer containing $100 \mathrm{~mm}$ for 1 $\mathrm{hr}$, or treated with chloroquine for $4 \mathrm{hr}$. Cells were fixed, and immunofluorescence for H2AX $\gamma$, ATMS1981p, and ATM(S1981) was assessed. $(B)$ Exponentially growing fibroblasts were incubated in hypotonic (100 $\mathrm{mm}$ or $50 \mathrm{~mm} \mathrm{NaCl})$ buffer for $1 \mathrm{hr}$ or in chloroquine for $4 \mathrm{hr}$. ATM was immunoprecipitated, and the levels of phosphorylated ATM, total ATM, and $\mathrm{H} 2 \mathrm{AX} \gamma$ were determined by immunoblotting. No cell death was observed in chloroquine or in the hypotonic conditions, and all cells recovered when returned to isotonic conditions. (C) Schematic model of ATM activation after irradiation. DNA strand breaks lead to an alteration of chromatin structures that induce intermolecular autophosphorylations of an ATM dimer on Ser-1981, and dissociation of the previously inert dimer. Active ATM monomers are then free to migrate to and phosphorylate substrates such as Nbs1 and p53. (Reprinted, with permission, from Bakkenist and Kastan 2003 [@ Nature Publishing Group].)

so completely after the introduction of so few DNA breaks. We postulated that one way in which ATM intermolecular autophosphorylation could be responding to DNA breaks at a distance was that the breaks were causing a change in higher-order chromatin structure through release of structural constraints by breaking the phosphodiester backbone. It was challenging to design experiments to test the hypothesis that ATM can be activated by alterations in higher-order chromatin structures, but we found that treatment of cells with either the topoisomerase inhibitor chloroquine or hypotonic buffer, both of which alter chromatin structure without generating detectable DNA breaks, induced phosphorylation of ATM at Ser-1981 (Fig. 2A,B) (Bakkenist and Kastan 2003).

More recently, we have observed that treatment of non- replicating $\mathrm{G}_{0}$ fibroblasts with the topoisomerase I poison, camptothecin, also results in ATM activation (C.J. Bakkenist and M.B. Kastan, unpubl.). In the absence of DNA replication, this drug treatment induces no DNA strand breaks, thus providing another example of ATM activation in the absence of DNA breakage. Immunofluorescence assays using the anti-S1981 phosphoantibody were also consistent with the model of ATM activation not requiring binding to DNA breaks. Following IR, immunostaining reveals a diffuse distribution of phosphoATM in the first few minutes after IR and then accumulation in foci, presumably at the sites of DNA breaks. However, following the hypotonic swelling or chloroquine treatments, phosphorylated ATM remains diffusely distributed throughout the nucleus, and no foci are ever seen. These results are all consistent with a model in which ATM activation is not dependent on direct binding of ATM to DNA double-strand breaks (DSBs) but instead results from changes in the structure of chromatin. Our current model of IR-induced ATM activation proposes that DNA breaks cause an alteration in some aspect of higher-order chromatin structure which in turn induces a conformational change in ATM that causes intermolecular autophosphorylation of Ser-1981 in the ATM dimer and consequent dimer dissociation (Fig. 2C). Active ATM monomers are then free to interact with and phosphorylate downstream targets of the signaling pathway, such as Nbs1 and p53.

Interestingly, in addition to Nbs1 protein being a substrate of ATM, the Mre11/Rad50/Nbs1 (MRN) complex appears to be quantitatively important for ATM activation following IR (but not following many other cellular stresses) (Carson et al. 2003; Mochan et al. 2003; Uziel et al. 2003; Kitagawa et al. 2004). How the MRN complexes influence ATM activation after IR remains to be elucidated, but this observation by itself does not necessarily mean that the ATM dimer goes to DNA breaks to get activated; MRN could be influencing the chromatin structure changes that occur following IR and thus influence ATM activation directly. Once activated, it is clear that MRN is important for recruiting the activated ATM to the DNA break (Kitagawa et al. 2004). The fact that other stresses, such as hypotonic swelling, do not require MRN for ATM activation also demonstrates that binding of ATM dimers to DNA breaks is not required for ATM activation.

Lee and Paull (2005) recently developed an elegant in vitro assay to assess ATM activation. Using a gradient analysis, they confirmed our model and the work of Unsal-Kucmaz and Sancar (2004) by demonstrating that ATM exists as a dimer and that activation associated with S1981 phosphorylation results in dimer dissociation. The major distinction between our model and the results described using this in vitro assay is that they concluded that S1981 phosphorylation was not required for ATM activation because the S1981A mutant was already active in vitro. Since we found that the S1981 mutant was active in vitro but had no cellular activity, it seems more appropriate to draw the conclusion that their in vitro model for testing ATM activation, dissociation, and activity, al- 
though elegant, does not accurately reproduce the in vivo situation for initiating ATM activation. It is likely that this results from an artifactual dissociation of the ATM dimer under these in vitro conditions, even in the absence of S1981 phosphorylation. The fact that there was plenty of ATM monomer in their preparations of S1981A mutant ATM would be consistent with this conclusion.

\section{PHOSPHORYLATION OF SUBSTRATES BY ATM}

When cells are irradiated, ATM is quickly activated, and it phosphorylates many substrates (Kastan and Lim 2000). Phosphorylation of $\mathrm{p} 53$, mdm2, and Chk2 activates the $\mathrm{G}_{1}$ checkpoint (Canman et al. 1998; Ahn et al. 2000; Matsuoka et al. 2000; Maya et al. 2001). Phosphorylation of Nbs1, FancD2, and Ser-1387 of Brca1 is required for the S-phase checkpoint (Lim et al. 2000; Taniguchi et al. 2002; Xu et al. 2002b), and phosphorylation of Rad17 and Ser-1423 of Brca1 is involved in the $\mathrm{G}_{2}$ checkpoint (Bao et al. 2001; Xu et al. 2002b). Recently, we identified SMC1 (structural maintenance of chromosomes 1) as an ATM substrate. We also identified two ATM phosphorylation sites on SMC1 and found that the phosphorylation of this substrate by ATM is required for the proper activation of the S-phase checkpoint (Kim et al. 2002; Yazdi et al. 2002). To identify which checkpoint and which target are important for determining cellular radiosensitivity, we mutated selected phosphorylation sites in the various proteins and were somewhat surprised to be able to demonstrate that neither the $G_{1}$ checkpoint, intra-S-phase checkpoint, nor early $\mathrm{G}_{2}$ checkpoint affected cellular survival after irradiation (Slichenmyer et al. 1993; Lim et al. 2000; Xu et al. 2002a,b). Interestingly, the only target of ATM in which mutation of the phosphorylation sites affected cellular radiosensitivity was SMC1 (Kim et al. 2002; Kitagawa et al. 2004). Subsequent comments focus just on the role of SMC1 and its phosphorylation in DNA damage responses.

\section{SMC1 IS AN ATM SUBSTRATE AND PARTICIPATES IN CELLULAR RESPONSES TO DNA DAMAGE}

SMC1 is a member of the structural maintenance of chromosomes family (Strunnikov and Jessberger 1999). It was originally identified in budding yeast as a component of the cohesin complex (Michaelis et al. 1997), where it exists as a heterodimer with $\mathrm{Smc} 3$ (Anderson et al. 2002; Haering et al. 2002). This SMC1-SMC3 heterodimer has also been identified in the RC-1 complex, which is a mammalian protein complex involved in DNA recombination (Jessberger et al. 1996; Stursberg et al. 1999). These findings provided the first suggestion that SMC1 might be involved in DNA recombination and DNA repair. Unexpectedly, we found that SMC1 was phosphorylated on two sites, Ser-957 and Ser-966, in response to IR in an ATM-dependent manner (Fig. 3A,B) (Kim et al. 2002; Yazdi et al. 2002). Both sites were also phosphorylated in response to ultraviolet (UV) light or
A

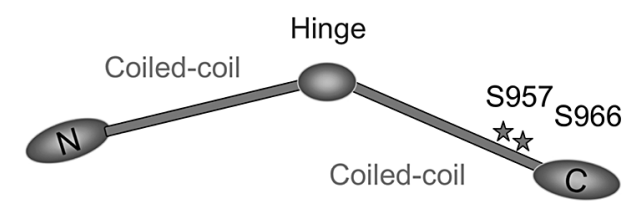

B

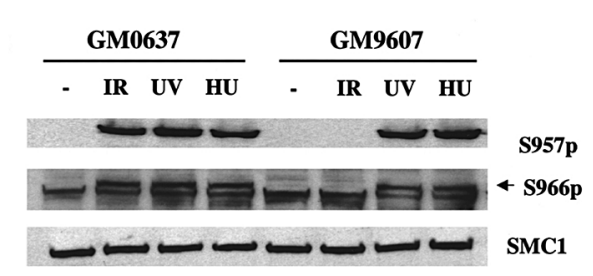

C

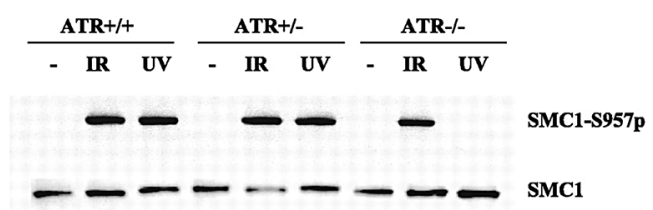

Figure 3. DNA damage-induced phosphorylation of Ser-957 and Ser-966 of SMC1. (A) Schematic representation of the domains of Smc1 illustrating the approximate location of the Ser957 and Ser-966 sites with the stars. (B) ATM-dependent and independent phosphorylation of SMC1 on Ser-957 and Ser-966. Immunoblot analyses using the two phosphoserine-specific antibodies (top and middle) or an anti-SMC1 antibody (K1; bottom) on SMC1 protein from normal (WT; GM0637) or A-T (GM9607) fibroblasts treated with ionizing irradiation (IR; 10 Gy, $2 \mathrm{hr}$ ), ultraviolet light (UV; $50 \mathrm{Jm}-2,2 \mathrm{hr}$ ) or hydroxyurea (HU; $1 \mathrm{mM}, 24 \mathrm{hr}$ ). The arrow (middle) points to the band specifically recognized by the antiphosphoserine 966 antibody (note a nonspecific band runs just below the S966-p band). (C) ATRdependent phosphorylation of S957 of SMC1. Immunoblot analyses using a phosphoserine-specific antibody against SMC1S957p or an anti-SMC1 antibody (K1; bottom) on SMC1 protein from $\mathrm{ATR}^{+/+}$(HCT116), $\mathrm{ATR}^{+1}$ (HCT116 flox), or $\mathrm{ATR}^{\prime}$ (HCT flox infected with AdCre virus) cells 30 min after treatment with $0.06 \mathrm{~J} / \mathrm{m} 2 \mathrm{UV}$ radiation. ( $B$, Reprinted with permission from Kim et al. 2002; $C$, reprinted, with permission, from Garg et al. 2004.)

hydroxyurea treatment in an ATM-independent manner (Kim et al. 2002); in this setting, the related kinase ATR was phosphorylating these same two serines (Fig. 3C) (Garg et al. 2004). Therefore, Ser-957 and Ser-966 are phosphorylated after virtually every type of DNA damage, suggesting that phosphorylation of SMC1 is important in many cellular stress responses.

Although we had previously investigated the functional significance of phosphorylation events in NBS1 or BRCA1 by complementing NBS1-deficient cells or BRCA1-deficient cells with phosphorylation-mutant constructs, we could not use this approach to study SMC1 phosphorylation because there are no SMC1-null cell lines available. To explore the physiological significance of SMC1 phosphorylation, we overexpressed recombi- 
nant SMC1 cDNA constructs in which Ser-957 and Ser966 were replaced with alanine residues such that DNAdamage-induced phosphorylation of those sites does not occur, and asked whether dominant-negative effects could be seen with overexpression of this ectopic mutant protein. Cells overexpressing mutant SMC1 exhibited increased radiosensitivity and an intra-S-phase checkpoint defect (Kim et al. 2002), thus implicating these phosphorylation events as determinants of these cellular endpoints.

\section{SMC1 IS "DOWNSTREAM" IN THE ATM SIGNALING PATHWAY}

We were surprised to find that radiation-induced phosphorylation of Ser-957 and Ser-966 in SMC1 did not occur in cells lacking full-length NBS1 or BRCA1 proteins and could be restored by complementation of these cells with full-length constructs of their respective mutant proteins (Kim et al. 2002). These effects did not result from a lack of ATM activation in the mutant cells, leading us to hypothesize that NBS1 and BRCA1 played a role in recruiting activated ATM to sites of DNA DSBs, and that once there, the activated ATM could phosphorylate SMC1. To test this theory, we analyzed the subcellular localization of phosphorylated ATM in cells lacking fulllength NBS1 or full-length BRCA1. In the presence of full-length NBS1 and BRCA1, phosphorylated ATM localized at the sites of DNA strand breaks after IR, but in the absence of either full-length protein, ATM was activated but remained diffusely distributed throughout the nucleus and did not form foci (Kitagawa et al. 2004). These observations suggested that NBS1 and BRCA1 are required for efficient recruitment and/or retention of activated ATM to the sites of DNA breaks, where the activated ATM can then phosphorylate substrates at the break, such as SMC1, resulting in proper activation of the DNA-damage-induced signaling pathway. Consistent with this hypothesis, Lee and Paull (2004) demonstrated that the MRN complex stabilizes the binding of ATM to substrates in vitro and that the MRN complex is required for in vitro binding of purified ATM to linearized DNA molecules immobilized on beads (Lee and Paull 2005).

The only insights we had at this point about the functional role of SMC1 phosphorylation had come from the studies overexpressing the phospho-mutant SMC1 constructs. To more effectively study the functional role of SMC1 phosphorylation, we generated mice and cells that contained normally regulated SMC1, but where the SMC1 protein could not be phosphorylated because of mutation of the target serines. In mouse fibroblasts containing Smc1S957AS966A, IR-induced phosphorylation of Atm, p53, Nbs1, and Brcal were all comparable to that seen in cells containing wild-type Smc1 (Fig. 4A) (Kitagawa et al. 2004). However, as expected, no phosphorylation of Smc1 was detectable (Fig. 4A). Despite the absence of phosphorylated Smc1 at the sites of DNA strand breaks, we easily detected foci containing activated (phosphorylated) Atm, H2AX $\gamma, \mathrm{Nbs} 1$, 53BP1, Chk2T68p, and Brcal (Fig. 5). Thus, SMC1 phosphory-

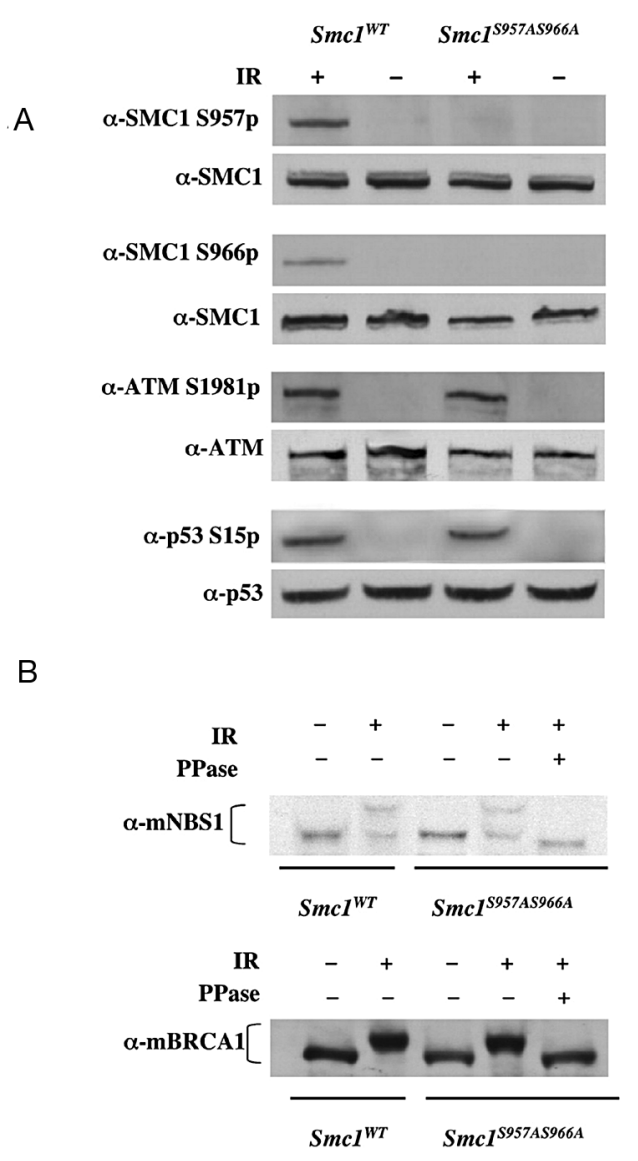

Figure 4. IR-induced phosphorylation of ATM and its target proteins in the absence of phosphorylation of SMC1. (A) Wildtype $(S m c 1 W T)$ or $S m c 1 S 957 A S 966 A$ immortalized mouse fibroblast cells were exposed to 0 Gy (IR-) or $10 \mathrm{~Gy}$ (IR+) of IR and cellular extracts were prepared $30 \mathrm{~min}$ later. Whole-cell lysates were subjected to immunoblotting with antibodies as indicated. $(B)$ Thirty minutes after exposure to $0(-)$ or $10(+)$ Gy of IR, whole-cell lysates were prepared from wild-type, or mutant knock-in fibroblasts were immunoblotted with anti-mouse Nbs1 or Brca1 antibodies. Duplicate samples of cell lysates from irradiated knock-in cells were treated with protein phosphatase $(\mathrm{PPase}+)$ prior to electrophoresis. (Reprinted, with permission, from Kitagawa et al. 2004.)

lation was dependent on ATM, Nbs1, and Brca1, but nothing in the pathway appeared to be dependent on the phosphorylation of SMC1, thus placing SMC1 phosphorylation at the bottom of the ATM DNA damage response pathway characterized to date (Kitagawa et al. 2004).

However, if we were to conclude that SMC1 phosphorylation played any critical role in this DNA damage response pathway, we needed to demonstrate that the cells containing unphosphorylatable SMC1 had some defect in DNA damage responses. Consistent with results from studies using human cells overexpressing SMC1 mutant proteins, we found that the Smc1S957AS966A cells both lacked the IR-induced intra-S-phase checkpoint (Fig. 6A) and were hypersensitive to DNA damaging agents such as IR and methylmethane sulfonate (MMS) (Fig. 6B,C). Furthermore, we found that these "knock-in" cells exhibited decreased removal of chromosome gaps and breaks 

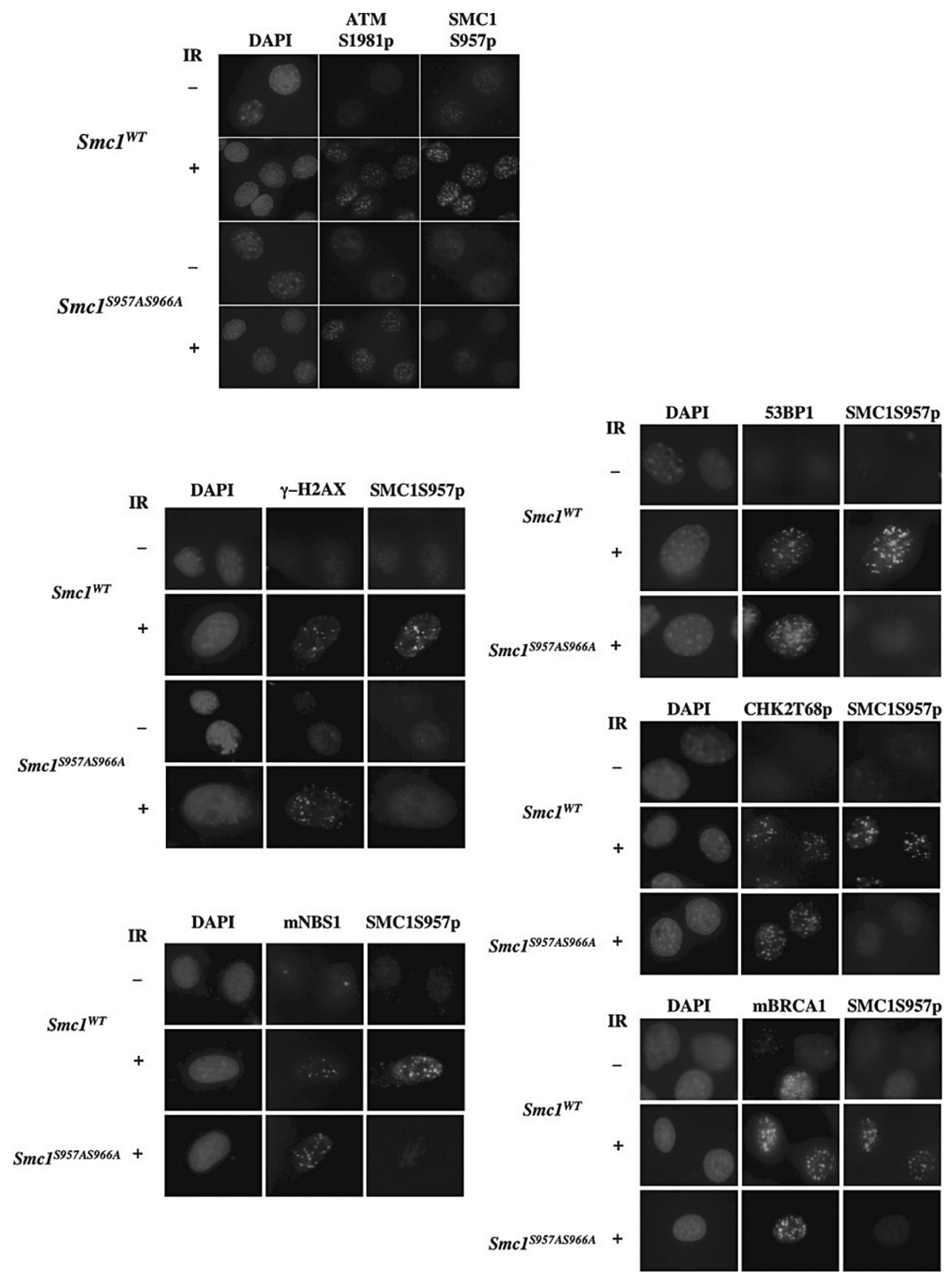

Figure 5. Smc1 phosphorylation is not required for IR-induced formation of foci containing phospho-Atm, H2AX, Nbs1, 53BP1, phosphorylated Chk2, or Brca1. Wild-type (Smc1WT) or Smc1 phosphorylation mutant knockin (Smc1S957AS966A) fibroblast cells were fixed with $4 \%$ paraformaldehyde $30 \mathrm{~min}$ after $0 \mathrm{~Gy}(-) 10 \mathrm{~Gy}(+)$ of IR, then subjected to immunofluorescence microscopy. $(A)$ Staining with antibodies recognizing phosphorylated Atm or Smc1. (B) Staining with antibodies recognizing H2AX, Nbs1, 53BP1, phosphorylated Chk2, Brca1, and phosphorylated Smc1. For costaining of phosphorylated Smc1 with Brca1, rabbit polyclonal antiSer957p antibody was used. (Reprinted, with permission, from Kitagawa et al. 2004.)

after IR, a phenotype shared with cells lacking ATM (Fig. 6D). This finding suggests that a DNA repair activity is reduced in Smc1S957AS966A cells, at least during the first hour after DNA breaks are introduced.

Phosphorylation of Smc1 at Ser-957 and Ser-966 thus appears to be a crucial downstream event in the DNAdamage-induced signaling pathway and may be required for proper activation of DNA repair activity to maintain optimal cell viability after DNA damage. As shown in the model we propose (Fig. 7), an ordered dependency of events occurs after the initiation of the DNA-damage-induced signaling pathway. Ionizing radiation introduces DNA breaks into genomic DNA, resulting in some highorder structural changes in chromatin. (This aspect is admittedly speculative but is most consistent with our data.) The chromatin change generates a signal that induces intermolecular autophosphorylation of ATM. The phosphorylated ATM monomer is then released from its inac- 
A
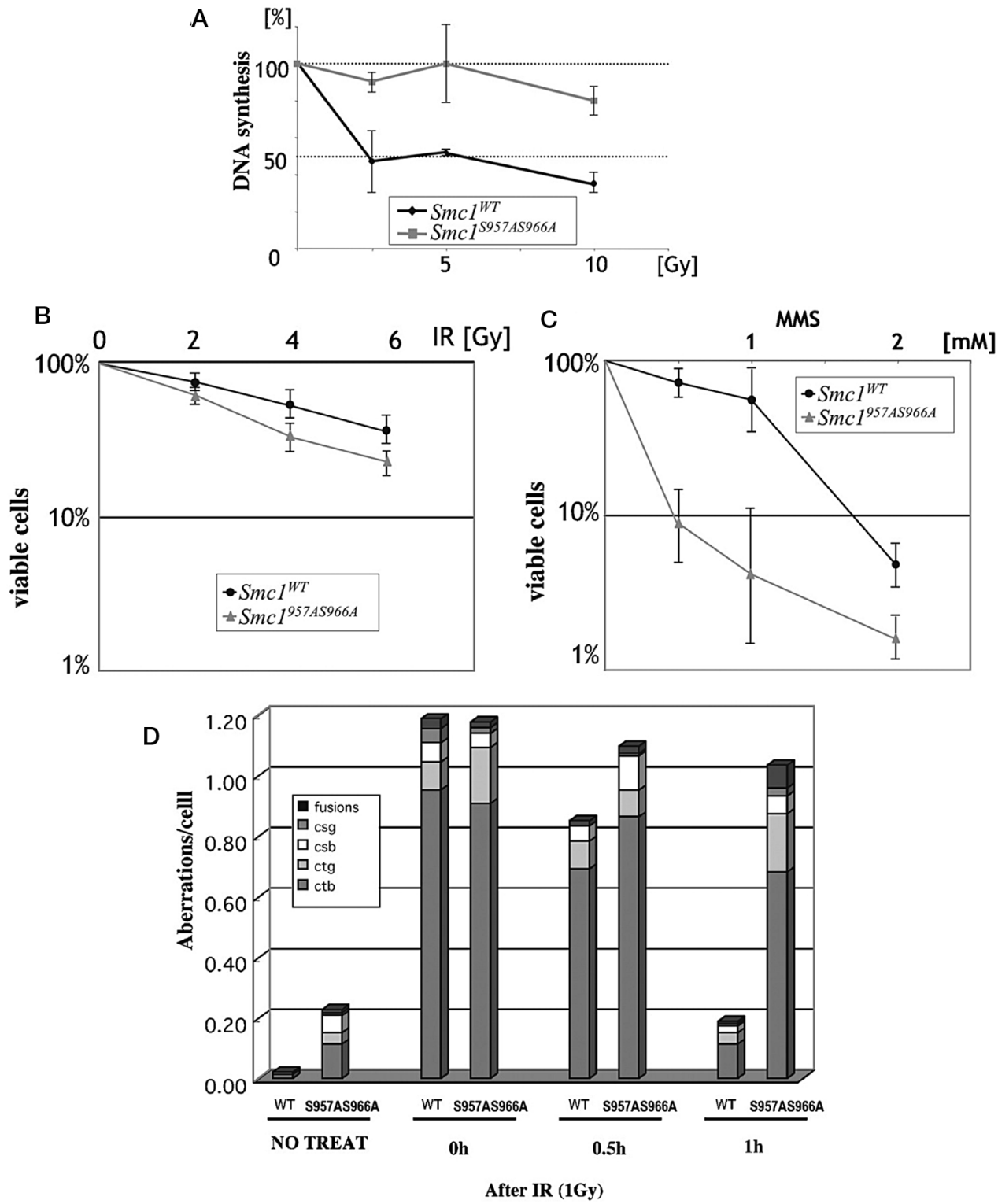

Figure 6. Radiation response abnormalities in Smc1S957AS966A cells. (A) Defect in the S-phase checkpoint. Mouse embryonic fibroblasts (MEFs) derived from wild-type (Smc1WT) or Smc1S957AS966A embryos were assessed for inhibition of DNA synthesis 30 min after exposure to indicated doses of IR. Error bars are average of triplicate samples. $(B)$ Increased sensitivity to IR. Wild-type or Smc1S957AS966A immortalized mouse fibroblast cells were plated in 6-well culture dishes and irradiated with the indicated doses of IR. Cell viability was assessed $72 \mathrm{hr}$ after treatment and is plotted as the percent viable cells relative to results for untreated control cultures. Each point represents the mean of three samples, with error bars showing standard deviation. $(C)$ Increased sensitivity to alkylating agents. Wild-type or Smcl mutant knockin immortalized mouse fibroblast cells were plated in 6-well culture dishes, and treated with methylmethane sulfonate (MMS) for $1 \mathrm{hr}$. Cell viability was assessed $72 \mathrm{hr}$ after treatment, and is plotted as the percent viable cells relative to results for untreated control cultures. Each point represents the mean of three samples, with error bars showing standard deviation. $(D)$ Defect in chromosomal repair after IR. MEFs derived from wild-type or Smc1S957AS966A embryos were irradiated with $1 \mathrm{~Gy}$ of IR, then treated with colcemid at indicated times after irradiation. Chromosome aberrations were scored in 100 metaphase spreads of each sample, and numbers of chromosome gaps (csg), chromosome breaks (csb), chromatid gaps (ctg), and chromatid breaks (ctb) per cell were charted. (Reprinted, with permission, from Kitagawa et al. 2004.)

tive dimer complex and phosphorylates nucleoplasmic substrates such as $\mathrm{p} 53$. At the same time, proteins migrate in a highly ordered manner to the DSB sites to form foci of protein complexes that include SMC1. The recruitment of previously activated ATM to the DSBs depends on the presence of NBS1 and BRCA1. Once the activated ATM binds to the focus complex, the ATM substrates can be phosphorylated. Phosphorylation of these proteins is required for proper activation of the DNA repair pathway and the cell cycle checkpoint pathways, and phosphorylation of SMC1 is specifically critical for cell survival and maintenance of optimal chromosomal stability. 

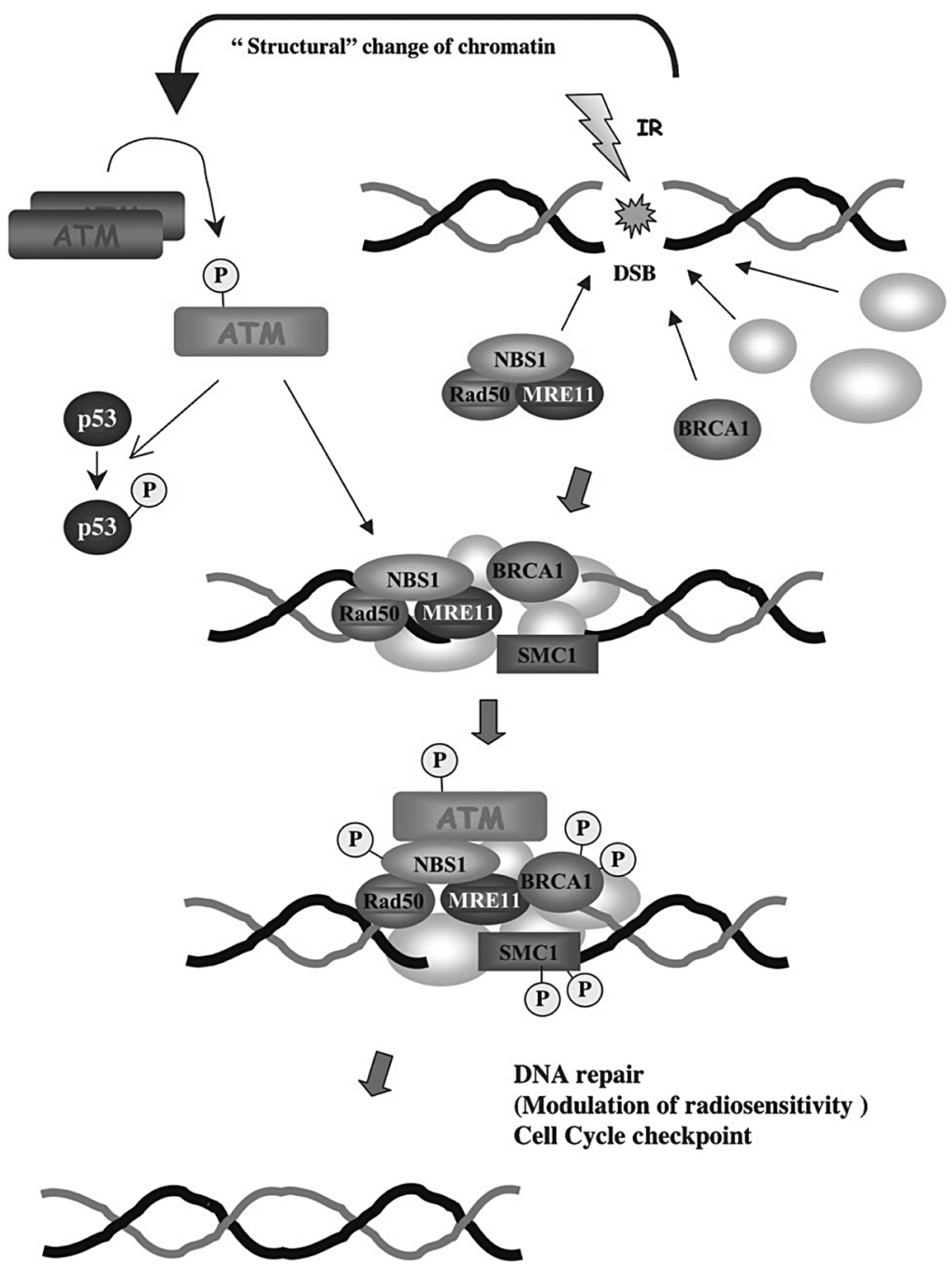

Figure 7. Proposed model for an IR-induced signaling pathway. Chromatin structure changes caused by DNA breakage or other mechanisms lead to intermolecular autophosphorylation of ATM dimers, resulting in release of phosphorylated and active ATM monomers. If DNA strand breaks are present, several proteins, including NBS1 and BRCA1, are recruited to the sites of the breaks independent of the ATM activation process. After activation, monomeric ATM can phosphorylate nucleoplasmic substrates, like p53, and if NBS1 and BRCA1 have localized to DNA breaks, activated ATM is recruited to the break. At the DNA break, activated ATM can phosphorylate substrates, including SMC1. The phosphorylation of SMC1 reduces chromosomal breakage and enhances cell survival. (Reprinted, with permission, from Kitagawa et al. 2004.)

\section{SMC1 AND MECHANISMS OF DNA REPAIR}

Although the results described above strongly implicate SMC1 and its phosphorylation in cellular responses to DNA damage and to DNA repair specifically, we have no insights yet as to the exact repair mechanisms that SMC1 might influence. Based on known repair mechanisms and characterized aspects of the cohesin complex, the following discussion speculates on potential roles for SMC1 in DNA repair processes. DNA DSBs can be repaired by either nonhomologous end-joining (NHEJ) or homologous recombination (HR). HR repair of DNA DSBs requires an intact DNA sequence homologous to the one needing repair to serve as a template. Because the sister chromatid would be the preferential template for repair, it has been suspected that the sister chromatid cohesin complex plays a role in efficient HR repair of DNA
DSBs, at least in yeast. Mutants of the cohesin proteins such as $s c c 1 /$ rad 21 in yeast are hypersensitive to $\gamma$-irradiation (Birkenbihl and Subramani 1992), and we showed that a hypomorphic mutation of $s m c 1$ causes yeast to be sensitive to various types of DNA damage (Kim et al. 2002). Sjogren and Nasmyth (2001) demonstrated defective DSB repair activity of cohesin mutants including $s c c 1, s c c 2$, and $s m c 1$ by using pulse-field gel electrophoresis to monitor induction and disappearance of DSBs in synchronized yeast cells. Their analysis revealed that cohesin proteins are specifically required for postreplicative HR repair of chromosomal DNA. In addition, two groups have demonstrated the recruitment of components of the cohesin complex to the sites of DNA DSBs generated by induction of $\mathrm{HO}$ endonuclease (Strom et al. 2004; Unal et al. 2004). Both groups used chromatin immunoprecipitation (ChIP) assays to show 
the accumulation of cohesin proteins at the single $\mathrm{HO}$ site when a break was introduced. The DNA-break-specific cohesin domain expanded to an approximately $100-\mathrm{kb}$ chromosomal region around the lesion, and the formation of that domain required the phosphorylation of $\mathrm{H} 2 \mathrm{AX} \gamma$.

The efficiency of the DNA DSB repair pathway, which is distinct from the HR repair pathway, also depends on Smc1. Schar et al. (2004) demonstrated that the loss of functional Smc1 reduced the efficiency of NHEJ repair in episomal DSBs and substantially increased the frequency of errors in the remaining end-joining activity in yeast. The $s m c 1$ hypomorphic mutant genetically interacted with $d n l 4$, a factor involved in the NHEJ pathway, and rad54, a factor required for the HR pathway. Smc1 negatively regulates the inhibitory effects of Rad52 and Rad54 on NHEJ activity. These genetic findings suggest that Smc1 coordinates the appropriate balance between the HR and NHEJ repair pathways. However, whether the role of Smc1 is distinct from that of other cohesin proteins and whether Smc1 always forms a heterodimer with Smc3 or is always a component of the cohesin complex remain unknown. Thus, fully functional $\mathrm{Smc1}$ is required for a proper DNA damage response in yeast, presumably as a component of the functional cohesin complex. Although the phosphorylation sites identified in human SMC1 are not conserved in budding yeast, a fundamental mechanism by which this ATM substrate contributes to the efficient repair of DNA DSBs could be conserved.

To study the molecular mechanism(s) underlying the phosphorylation events at Ser-957 and Ser-966 of human SMC1, it was important to determine whether phosphorylation at those sites affects the cohesion function of the sister chromatid. SMC proteins have globular domains at their amino and carboxyl termini, which are separated by two long stretches of coiled-coil domains. The coiled-coil domains are connected to the third globular domain, which is called the "hinge" due to its flexible feature (Fig. 3A). SMC proteins form V-shaped molecules by dimerizing via their central hinge domain. In eukaryotes, different SMC proteins heterodimerize in specific pairs: Smc1-Smc3, Smc2-Smc4, and Smc5-Smc6 (Hirano and Hirano 2002; Haering and Nasmyth 2003). In the cohesin complex, Smc1 and Smc3 associate with non-SMC subunits $\operatorname{Scc} 1 / \operatorname{Rad} 21$ and $\mathrm{Scc} 3$ (in humans, SA1 and SA2 replace $\operatorname{Scc} 3)$. Scc1/Rad21 binds to the head of Smc3 via its amino-terminal domain and to that of Smc1 via its carboxy-terminal domain (Haering et al. 2002). Scc3 associates with $\mathrm{Smc} 1-\mathrm{Smc} 3$ by interacting with the central domain of Scc1/Rad21. SMC1 phosphorylation sites, Ser-957 and Ser-966, are located at the carboxy-terminal end of the coiled-coil domain, near the carboxy-terminal globular domain (Fig. 3A). IR-induced phosphorylation of those sites does not affect the interaction of SMC1 with SMC3 or RAD21 (Kim et al. 2002; R. Kitagawa and M.B. Kastan, unpubl.). Consistent with the finding that the phosphorylation status of SMC1 did not affect the formation of the cohesin complex, mouse Smc1S957AS966A cells do not exhibit any noticeable cohesion defects (e.g., premature sister chromatid separation or mitotic-checkpoint-dependent delay of mitosis)
(R. Kitagawa and M.B. Kastan, unpubl.).

Although the phosphorylation of SMC1 has no noticeable effect on the formation of the cohesin complex, it may affect the association of cohesin with chromosomes. Based on a recent finding that TEV-protease-dependent cleavage of the Smc3 coiled-coil domain triggers the dissociation of cohesion from chromosomes, it has been proposed that cohesin forms a ring-like structure that encircles two sister chromatids (Gruber et al. 2003). The ATPase activity of the head domain in Smc proteins is required for its binding to $\operatorname{Scc} 1 / \operatorname{Rad} 21$ and results in the formation of the cohesin ring, which enables cohesin to associate with chromosomes (Arumugam et al. 2003; Weitzer et al. 2003). The cohesin ring model in which the association of cohesin with chromosomes is possibly topological in nature rather than protein-DNA binding reasonably explains why the separase-mediated cleavage of Scc1/Rad21 at metaphase-anaphase transition is sufficient to trigger the dissociation of the cohesin complex from chromosomes. Furthermore, topological association of the cohesin ring with chromatin would allow cohesin considerable mobility. This flexibility in movement explains the instantaneous formation of DSB-specific cohesin complexes. Again, Smc1S957AS966A cells do not exhibit any noticeable cohesin defects during the normal cell cycle progression, a finding that suggests that the phosphorylation of SMC1 does not affect the association of the cohesin complex with chromosomes at the cohesions. However, assuming that the formation of DSBspecific cohesin complex is a common event in eukaryotic cells, phosphorylation status of SMC1 might specifically affect the establishment or stability of the complex at the sites of DSBs.

In addition to being a component of the cohesin complex, SMC1 is a DNA-binding protein (Akhmedov et al. 1998). Specific binding of the carboxy-terminal region of Smc1 to highly structured DNA (e.g., stem-loop-structured DNA or DNA-containing AT-rich sequences) has been demonstrated in vitro by using purified recombinant protein. As described above, SMC1 is phosphorylated at two serine residues in the carboxy-terminal end of the coiled-coil domain. Therefore, its phosphorylation status may affect the DNA-binding activity of SMC1 via its carboxy-terminal domain. Although the physiological role of this DNA-binding activity of SMC1 has not been elucidated, it may be required for the DNA damage response.

Zheng et al. (2000) showed that the DNA-binding activity of Smc1 affinity-purified from yeast cells was enhanced when human Hec1 and Rb protein were artificially expressed in yeast cells. Human SMC1 also coimmunoprecipitates with $\mathrm{Rb}$ and human $\mathrm{Hec} 1$ from human T24 cell lysate. Thus, the interaction between SMC1 and DNA is modulated by SMC1's association with noncohesin proteins. Phosphorylation of SMC1 might affect the association of the cohesin complex with chromosomes by altering the interaction between the complex and other proteins such as Hecl.

Another attractive model for the mechanism by which phosphorylation of SMC1 plays a role in DNA damage 
response is that phosphorylation affects the binding of SMC1 to proteins required for DNA repair activities (e.g., DNA-modifying enzymes) and plays a role in recruitment of those enzymes to the sites of DNA lesions. Castano et al. (1996) identified Trf4, a member of DNA polymerase sigma protein family, as an SMC1 interactor and showed its role in DNA damage response. Trf4 exhibits DNA polymerase activity (Wang et al. 2000) and RNA polyadenylase activity (Saitoh et al. 2002). Trf4 may be recruited to the sites of DNA damage via its interaction with Smc1, and that interaction may be regulated by the phosphorylation status of Smc1.

\section{CONCLUSIONS}

The Atm protein kinase is a central mediator of cellular responses to aberrant introduction of DNA strand breaks. When such breaks are introduced in cells, Atm is rapidly activated by intermolecular phosphorylation at Ser-1981, which causes dissociation of the Atm homodimer. The activated Atm is then free to circulate in the cell and phosphorylate substrates, such as p53 in the nucleoplasm. Fulllength $\mathrm{Nbs} 1$ and Brcal proteins are required to attract or retain the activated Atm at the site of the DNA break. Once Atm is brought to the break, it can phosphorylate substrates at the break, with SMC1 appearing to be a critical substrate for maximizing cell survival and minimizing chromosomal aberrations. Although the phosphorylation of SMC1 does not significantly affect the formation of the cohesin complex, it may control the association of this complex with a particular structure of chromosomes or other repair proteins in cells after DNA damage is introduced. Identifying other proteins or DNA structures that directly associate with SMC1 and analyzing whether the phosphorylation status of SMC1 affects complex formation with those factors will help us further elucidate the molecular mechanism by which phosphorylation of SMC1 mediates the DNA repair pathway. The use of Smc1S957AS966A cells will be a powerful and invaluable reagent in this pursuit.

\section{REFERENCES}

Ahn J.Y., Schwarz J.K., Piwnica-Worms H., and Canman C.E. 2000. Threonine 68 phosphorylation by ataxia telangiectasia mutated is required for efficient activation of Chk2 in response to ionizing radiation. Cancer Res. 60: 5934.

Akhmedov A.T., Frei C., Tsai-Pflugfelder M., Kemper B., Gasser S.M., and Jessberger R. 1998. Structural maintenance of chromosomes protein C-terminal domains bind preferentially to DNA with secondary structure. J. Biol. Chem. 273: 24088.

Anderson D.E., Losada A., Erickson H.P., and Hirano T. 2002. Condensin and cohesin display different arm conformations with characteristic hinge angles. J. Cell Biol. 156: 419.

Arumugam P., Gruber S., Tanaka K., Haering C.H., Mechtler K., and Nasmyth K. 2003. ATP hydrolysis is required for cohesin's association with chromosomes. Curr. Biol. 13: 1941.

Bakkenist C.J. and Kastan M.B. 2003. DNA damage activates ATM through intermolecular autophosphorylation and dimer dissociation. Nature 421: 499.

Banin S., Moyal L., Shieh S., Taya Y., Anderson C.W., Chessa L., Smorodinsky N.I., Prives C., Reiss Y., Shiloh Y., and Ziv
Y. 1998. Enhanced phosphorylation of p53 by ATM in response to DNA damage. Science 281: 1674.

Bao S., Tibbetts R.S., Brumbaugh K.M., Fang Y., Richardson D.A., Ali A., Chen S.M., Abraham R.T., and Wang X.F. 2001. ATR/ATM-mediated phosphorylation of human Rad17 is required for genotoxic stress responses. Nature 411: 969.

Birkenbihl R.P. and Subramani S. 1992. Cloning and characterization of rad21 an essential gene of Schizosaccharomyces pombe involved in DNA double-strand-break repair. Nucleic Acids Res. 20: 6605.

Bosotti R., Isacchi A., and Sonnhammer E.L. 2000. FAT: A novel domain in PIK-related kinases. Trends Biochem. Sci. 25: 225.

Canman C.E., Lim D.S., Cimprich K.A., Taya Y., Tamai K., Sakaguchi K., Appella E., Kastan M.B., and Siliciano J.D. 1998. Activation of the ATM kinase by ionizing radiation and phosphorylation of p53. Science 281: 1677.

Carson C.T., Schwartz R.A., Stracker T.H., Lilley C.E., Lee D.V., and Weitzman M.D. 2003. The Mre11 complex is required for ATM activation and the $\mathrm{G} 2 / \mathrm{M}$ checkpoint. EMBO J. 22: 6610.

Castano I.B., Brzoska P.M., Sadoff B.U., Chen H., and Christman M.F. 1996. Mitotic chromosome condensation in the rDNA requires TRF4 and DNA topoisomerase I in Saccharomyces cerevisiae. Genes Dev. 10: 2564.

Garg R., Callens S., Lim D.S., Canman C.E., Kastan M.B., and $\mathrm{Xu}$ B. 2004. Chromatin association of rad17 is required for an ataxia telangiectasia and rad-related kinase-mediated S-phase checkpoint in response to low-dose ultraviolet radiation. Mol. Cancer Res. 2: 362.

Gruber S., Haering C.H., and Nasmyth K. 2003. Chromosomal cohesin forms a ring. Cell 112: 765.

Haering C.H. and Nasmyth K. 2003. Building and breaking bridges between sister chromatids. Bioessays 25: 1178 .

Haering C.H., Lowe J., Hochwagen A., and Nasmyth K. 2002. Molecular architecture of SMC proteins and the yeast cohesin complex. Mol. Cell 9: 773.

Hirano M. and Hirano T. 2002. Hinge-mediated dimerization of SMC protein is essential for its dynamic interaction with DNA. EMBO J. 21: 5733.

Jessberger R., Riwar B., Baechtold H., and Akhmedov A.T. 1996. SMC proteins constitute two subunits of the mammalian recombination complex RC-1. EMBO J. 15: 4061.

Kastan M.B. and Lim D.S. 2000. The many substrates and functions of ATM. Nat. Rev. Mol. Cell. Biol. 1: 179.

Kim S.T., Xu B., and Kastan M.B. 2002. Involvement of the cohesin protein, Smc1, in Atm-dependent and independent responses to DNA damage. Genes Dev. 16: 560 .

Kim S.T., Lim D.S., Canman C.E., and Kastan M.B. 1999. Substrate specificities and identification of putative substrates of ATM kinase family members. J. Biol. Chem. 274: 37538.

Kitagawa R., Bakkenist C.J., McKinnon P.J., and Kastan M.B. 2004. Phosphorylation of SMC1 is a critical downstream event in the ATM-NBS1-BRCA1 pathway. Genes Dev. 18: 1423.

Lee J.H. and Paull T.T. 2004. Direct activation of the ATM protein kinase by the Mre11/Rad50/Nbs1 complex. Science 304: 93.

. 2005. ATM activation by DNA double-strand breaks through the Mre11-Rad50-Nbs1 complex. Science 308: 551.

Lim D.S., Kim S.T., Xu B., Maser R.S., Lin J., Petrini J.H., and Kastan M.B. 2000. ATM phosphorylates p95/nbs1 in an Sphase checkpoint pathway. Nature 404: 613.

Matsuoka S., Rotman G., Ogawa A., Shiloh Y., Tamai K., and Elledge S.J. 2000. Ataxia telangiectasia-mutated phosphorylates Chk2 in vivo and in vitro. Proc. Natl. Acad. Sci. 97: 10389.

Maya R., Balass M., Kim S.T., Shkedy D., Leal J.F., Shifman O., Moas M., Buschmann T., Ronai Z., Shiloh Y., Kastan M.B., Katzir E., and Oren M. 2001. ATM-dependent phosphorylation of Mdm2 on serine 395: Role in p53 activation by DNA damage. Genes Dev. 15: 1067.

Michaelis C., Ciosk R., and Nasmyth K. 1997. Cohesins: Chromosomal proteins that prevent premature separation of sister 
chromatids. Cell 91: 35

Mochan T.A., Venere M., DiTullio R.A., Jr., and Halazonetis T.D. 2003. 53BP1 and NFBD1/MDC1-Nbs1 function in parallel interacting pathways activating ataxia-telangiectasia mutated (ATM) in response to DNA damage. Cancer Res. 63: 8586.

Saitoh S., Chabes A., McDonald W.H., Thelander L., Yates J.R., and Russell P. 2002. Cid13 is a cytoplasmic poly(A) polymerase that regulates ribonucleotide reductase mRNA. Cell 109: 563.

Savitsky K., Bar-Shira A., Gilad S., Rotman G., Ziv Y., Vanagaite L., Tagle D.A., Smith S., Uziel T., and Sfez S., et al. 1995. A single ataxia telangiectasia gene with a product similar to PI-3 kinase. Science 268: 1749.

Schar P., Fasi M., and Jessberger R. 2004. SMC1 coordinates DNA double-strand break repair pathways. Nucleic Acids Res. 32: 3921.

Shiloh Y. and Kastan M.B. 2001. ATM: Genome stability, neuronal development, and cancer cross paths. Adv. Cancer Res. 83: 209.

Sjogren C. and Nasmyth K. 2001. Sister chromatid cohesion is required for postreplicative double-strand break repair in Saccharomyces cerevisiae. Curr. Biol. 11: 991.

Slichenmyer W.J., Nelson W.G., Slebos R.J., and Kastan M.B. 1993. Loss of a p53-associated G1 checkpoint does not decrease cell survival following DNA damage. Cancer Res. 53: 4164.

Strom L., Lindroos H.B., Shirahige K., and Sjogren C. 2004. Postreplicative recruitment of cohesin to double-strand breaks is required for DNA repair. Mol. Cell 16: 1003

Strunnikov A.V. and Jessberger R. 1999. Structural maintenance of chromosomes (SMC) proteins: Conserved molecular properties for multiple biological functions. Eur. J. Biochem. 263: 6 .

Stursberg S., Riwar B., and Jessberger R. 1999. Cloning and characterization of mammalian SMC1 and SMC3 genes and proteins, components of the DNA recombination complexes RC-1. Gene 228: 1.

Taniguchi T., Garcia-Higuera I., Xu B., Andreassen P.R., Gregory R.C., Kim S.T., Lane W.S., Kastan M.B., and D'Andrea A.D. 2002. Convergence of the fanconi anemia and ataxia telangiectasia signaling pathways. Cell 109: 459.

Unal E., Arbel-Eden A., Sattler U., Shroff R., Lichten M., Haber J.E., and Koshland D. 2004. DNA damage response pathway uses histone modification to assemble a double-strand breakspecific cohesin domain. Mol. Cell 16: 991.

Unsal-Kacmaz K. and Sancar A. 2004. Quaternary structure of ATR and effects of ATRIP and replication protein A on its DNA binding and kinase activities. Mol. Cell. Biol. 24: 1292.

Uziel T., Lerenthal Y., Moyal L., Andegeko Y., Mittelman L., and Shiloh Y. 2003. Requirement of the MRN complex for ATM activation by DNA damage. EMBO J. 22: 5612 .

Wang Z., Castano I.B., De Las Penas A., Adams C., and Christman M.F. 2000. Pol kappa: A DNA polymerase required for sister chromatid cohesion. Science 289: 774.

Weitzer S., Lehane C., and Uhlmann F. 2003. A model for ATP hydrolysis-dependent binding of cohesin to DNA. Curr. Biol. 13: 1930.

Xu B., Kim S.T., Lim D.S., and Kastan M.B. 2002a. Two molecularly distinct $\mathrm{G}(2) / \mathrm{M}$ checkpoints are induced by ionizing irradiation. Mol. Cell. Biol. 22: 1049.

Xu B., O'Donnell A.H., Kim S.T., and Kastan M.B. 2002b. Phosphorylation of serine 1387 in Brcal is specifically required for the Atm-mediated S-phase checkpoint after ionizing irradiation. Cancer Res. 62: 4588.

Yazdi P.T., Wang Y., Zhao S., Patel N., Lee E.Y., and Qin J. 2002. SMC1 is a downstream effector in the ATM/NBS1 branch of the human S-phase checkpoint. Genes Dev. 16: 571.

Zheng L., Chen Y., Riley D.J., Chen P.L., and Lee W.H. 2000. Retinoblastoma protein enhances the fidelity of chromosome segregation mediated by hsHec1p. Mol. Cell. Biol. 20: 3529 . 


\section{$\$_{\text {SSH\& }}^{\infty} \mathrm{CS}$ Cold Spring Harbor Symposia SYMPOSIA on Quantitative Biology}

\section{The ATM-dependent DNA Damage Signaling Pathway}

R. KITAGAWA and M.B. KASTAN

Cold Spring Harb Symp Quant Biol 2005 70: 99-109

Access the most recent version at doi:10.1101/sqb.2005.70.002

References This article cites 48 articles, 26 of which can be accessed free at: http://symposium.cshlp.org/content/70/99.full.html\#ref-list-1

\section{License}

Email Alerting Receive free email alerts when new articles cite this article - sign up in Service the box at the top right corner of the article or click here. 